Revue d'histoire de l'Amérique française

REVUE D.HISTOIRE DE L'AMÉRIQUE FRANÇAISE

\title{
Le gouvernement responsable : 1848-1948
}

\section{Jean-Pierre Houle}

Volume 1, numéro 2, septembre 1947

URI : https://id.erudit.org/iderudit/801365ar

DOI : https://doi.org/10.7202/801365ar

Aller au sommaire du numéro

Éditeur(s)

Institut d'histoire de l'Amérique française

ISSN

0035-2357 (imprimé)

1492-1383 (numérique)

Découvrir la revue

Citer cet article

Houle, J.-P. (1947). Le gouvernement responsable : 1848-1948. Revue d'histoire de l'Amérique française, 1(2), 166-170. https://doi.org/10.7202/801365ar d'utilisation que vous pouvez consulter en ligne.

https://apropos.erudit.org/fr/usagers/politique-dutilisation/ 


\section{LE GOUVERNEMENT RESPONSABLE: 1848-1948}

$$
\text { - I - }
$$

Point DE DÉPART

La longue pratique d'un principe constitutionnel en estompe, aux yeux de la foule, l'importance intrinsèque et rejette dans l'oubli les luttes souvent très longues, toujours âpres, qui ont conduit à la reconnaissance, de jure et de facto, de ce principe devenu désormais un élément essentiel de la vie politique.

L'électeur souverain, ce produit d'une lente évolution historique, s'étonnerait peut-être qu'on lui rappelât une époque pendant laquelle ses représentants, ses commis aux fonctions législatives, n'exerçaient qu'un contrôle très lâche sur les décisions du pouvoir exécutif. Et pourtant, il en fut ainsi, au Canada, pendant un demi-siècle: - du mois de juin 1791 alors que le Parlement impérial introduisait dans la colonie le régime représentatif jusqu'au mois de mars 1848 qui marque la victoire définitive du principe de la responsabilité ministérielle. J'estime que cette période est la plus chargée de notre histoire, mais aussi par certains côtés, la plus belle; certainement la plus féconde. Elle est faite d'une lutte conduite par des hommes libres et qui se voulaient tels, doués d'un sens politique étonnant et dont on peut regretter l'aberration chez leurs descendants.

Mon intention n'est pas de retracer toutes les péripéties de cett lutte, ni d'en reconstituer tous les incidents. Je suppose connus de mes lecteurs, les faits essentiels. Bien que tout se tienne et se compénètre, puisque l'histoire c'est la vie, je m'en tiendrai, au moins pour la première partie de ce travail, à la ligne constitutionnelle.

$$
*^{*} *
$$

Le 10 juin 1791, la sanction royale était donnée à ce que l'on appelle l'Acte Constitutionnel. Où en étions-nous à cette date? 
Pourquoi un nouveau régime? Ce qui demeure, et l'élément nouveau? Politiquement et économiquement, le Canada est, depuis 1763, une "Crown Colony " c'est-à-dire un agent de production et de consommation destiné à alimenter la métropole en matières premières et à absorber les produits ouvrés de celle-ci. La dépendance économique avec la métropole est absolue. La dépendance politique ne l'est pas moins et elle est assurée par un gouverneur général, sorte de proconsul, comptable et responsable de ses faits et gestes devant le Parlement impérial uniquement. C'est là la conception coloniale du XVIIIIe siècle.

Au Canada, le problème dépasse les cadres trop étroits d'une politique mercantile. L'Angleterre a reçu une colonie française et catholique, dotée de tous les caractères d'une véritable nationalité, possédant ses institutions sociales et économiques et appartenant par l'origine ethnique de ses habitants, à l'une des plus grandes civilisations. Après une tentative d'assimilation brutale qui se révèle très tôt, vaine et erronnée; sous la pression d'événements extérieurs et sur l'avis des gouverneurs; malgré les marchands de Londres et les trafiquants de Québec et de Montréal, les conseillers de Sa Majesté s'inclinent devant un fait: l'existence d'un groupe national évolué, français et catholique. Le droit exprimé dans les capitulations et autres documents politiques, tels que le Placart d'Amherst, commandait, en plus, cette attitude. Lois civiles françaises rétablies, libre exercice de la religion romaine reconnue. Le texte de l'Acte de Québec comporte, il est vrai, une restriction d'importance, mais le législateur anglais ne pouvait pas ne pas l'inscrire. On sait d'ailleurs comment cette sorte de restriction peut être tournée et comment elle l'a été.

Sujets britanniques, les Canadiens obtiennent une reconnaissance officielle de leur droit à rester Français et catholiques. C'est tellement là le sens de l'Acte de Québec que celui-ci a alors été jugé - et il l'est encore par des historiens anglo-saxons - comme une erreur et comme une source de division.

Quels motifs animaient le législateur impérial? La question ne se pose pas ici. Disons simplement et parce que la même explication vaut pour 1791, - qu'il n'y a là, ni machiavélisme, ni naïveté. Réalisme d'une part, méconnaissance de l'importance coloniale du Canada, d'autre part. 
Vers 1780 , on se rend compte que la nouvelle politique ne produit pas les effets escomptés. La Révolution américaine, le fanatisme de la minorité, les agissements des hauts tonctionnaires limitent la portée de l'Acte de Québec. On aboutit à une impasse d'où l'on tentera de sortir par l'Acte Constitutionnel de 1791.

Notons tout de suite et pour n'avoir pas à y revenir, que l'Acte de 1791 n'enlève rien aux garanties déjà accordées par l'Acte de 1774 quant aux lois civiles et à la religion. Sur le terrain national, c'est une nouvelle affirmation de nos droits. Ce sont les cadres politiques et administratifs qui sont modifiés et de façon hardie, reconnaissons-le. Le territoire est scindé en deux parties afin de permettre à chaque groupe ethnique de se développer selon ses exigences. Ici encore, je néglige l'examen des motifs du législateur. Je note simplement que dans sa pensée, il ne pouvait s'agir là d'une solution définitive; l'espoir de fusionner les deux éléments n'était pas abandonné. Comment y parviendrait-on? Pour l'instant on l'ignore et à vrai dire on ne s'en préoccupe pas. Wait and see, c'est un principe politique.

Voici maintenant les nouveaux cadres:

a) le gouverneur, dont le rôle demeure le même - il règne et gouverne.

b) Un conseil exécutif et un conseil législatif dont les membres sont nommés.

c) Une Assemblée législative élue. Cette Assemblée était accordée à la demande de la minorité; les nôtres pour une part, y étaient hostiles et avec elle ils plongeaient dans l'inconnu puisqu'ils ignoraient tout des institutions parlementaires et que leur éducation politique les portait plutôt à les juger défavorablement. Au début, leur ignorance leur fera commettre des faux pas. A première vue, c'est là le seul défaut de la nouvelle constitution; défaut minime puisque très tôt, nos parlementaires, qui dans leur nouveau rôle n'ont pas abdiqué leur logique française, iront jusqu'à la conclusion des prémisses posées: ce que le législateur impérial n'avait certes pas prévu. Il est vrai qu'ils y apporteront en plus, leur nervosité, française elle aussi. Mais poussons davantage l'examen, sans quoi il n'y a rien à comprendre à la lutte qui va bientôt s'engager ou, ce qui est plus grave, on risque d'en fausser la direction.

Les membres des conseils ne sont pas élus, mais nommés par la Couronne et ils seront recrutés en majorité parmi la minorité. Quel- 
ques-uns de ces messieurs, ajoutons-le, siègent au Conseil législatif et à l'Assemblee; d'autres sont aussi magistrats; des membres de l'Exécutif sont en même temps des hauts fonctionnaires; un trop grand nombre enfin sont des adversaires de la majorité. Tous les gouverneurs, et certains avec une précipitation qui éclaire brutalement la scène politique, tomberont dans les filets de cette clique qui entretient à Londres des intelligences nombreuses et zélées. Le nouveau régime n'est donc représentatif que par l'Assemblée. Cela suffit puisque inévitablement celle-ci sera déléguée par la majoritée Mais attention! Rien dans la loi de 1791 qui détermine de façon précise les pouvoirs de chaque corps; plus, aucun mécanisme prévu pour les articuler, aucune règle pour maintenir l'équilibre. Voilà le vice constitutionnel: on introduit le régime représentatif mais on le prive des moyens de jouer à fond; on prétend offrir à la majorité un instrument qui lui permette de travailler à la réalisation de ses justes aspirations, mais on la prive des moyens de s'en servir. En réalité, on revient à l'époque antérieure, au gouvernement par une minorité. Enfin, au-dessus des Conseils et de l'Assemblée, le gouverneur, qui détient et exerce la plénitude du pouvoir.

Est-ce à dire que le parlement impérial retire d'une main ce qu'il accorde de l'autre? Encore que ce geste ne puisse scandaliser que les naifs, je n'y vois pour ma part aucun machiavélisme, je le répète. Le législateur anglais n'éprouve pas de répulsion envers les contradictions et il compte sur le temps pour les réduire. Il y a plus cependant: 10 la politique coloniale telle qu'elle existe alors et conformément au but qu'elle poursuit, ne peut permettre qu'une colonie échappe au contrôle sévère de la métropole. Or accorder le régime représentatif et en autoriser le libre jeu, c'est se condamner à relâcher puis à perdre ce contrôle. Cela est si évident que lors des discussions provoquées par le rapport Durham, les plus clairvoyants des politiques anglais saisiront la menace et affirmeront que doter les colonies du principe de la responsabilité ministérielle - principe commandé par les institutions parlementaires - c'est se résigner à l'indépendance des colonies; 20 la métropole est alors elle-même en pleine crise constitutionnelle.

Mais la logique de l'histoire l'emporte parfois sur une politique qui s'appuie uniquement sur le temps et qui n'est soucieuse que des seuls intérêts matériels. Instaurer un régime représentatif et en remet- 
tre la direction à une minorité, cela peut paraître expédient et habile, mais cela conduira à une impasse avec d'autant plus de rapidité et de frais que l'autorité s'entêtera dans une attitude constitutionnellement absurde; la minorité pour sa part, s'ingéniera à fausser de plus en plus l'appareil législatif; la majorité, fermement appuyée sur ses droits, trouvera des hommes habiles à profiter de chaque circonstance, pour enrayer la manœuvre du pouvoir. Vient un moment que les passions recouvrent tout et risquent d'emporter les fondements mêmes de l'édifice.

Dans la prochaine livraison, nous analyserons les grandes étapes de la marche vers le gouvernement responsable.

Jean-Pierre Houle professeur d l'Institut d'Histoire, Faculté des Lettres, Université de Montréal.

SUR LeS PISTES De LA RevUt

La Revue d'Histoire de l'Amérique française suscite déjà des collaborations assez inattendues. Dans notre première livraison, M. J.-Alfred Perrault publiait, sur son ancêtre, un article intitulé comme suit: Nicolas Perrot a-t-il tenté d'empoisonner Cavelier de la Salle? Il offrait, en même temps, son abondante documentation aux historiens qui voudraient écrire l'histoire du grand voyageur. L'article, de par l'obligeance de M. Gustave Lanctot, archiviste d'Ottawa, tomba entre les mains du révérend L. Short, ancien curé de la paroisse Saint-François-Xavier (St. Francis, Wisconsin), qui n'est autre que l'ancienne mission des Jésuites en cette région. Le révérend Short travaille à un ouvrage sur le Père Allouez et Nicolas Perrot. Il prétend y élucider quelques points restés obscurs des premières découvertes du Mississipi. L'ouvrage parâtrait en février prochain.

Le révérend, mis sur une bonne piste, se rendit récemment a Outremont (Québec, Canada). M. J.-Alfred Perrault lui donna gracieusement accès à sa documentation. Ensemble, l'historien et le descendant de Perrot entreprirent le pèlerinage à Becancour pour y découvrir l'emplacement de l'ancienne ferme de l'ancêtre. L'abbé Short chanta, dans l'église de Bécancour, servie par M. J.-Alfred Perrault, une messe de requiem pour le repos de l'âme du voyageur. L'ancien curé de Saint-François-Xavier, Wisconsin, garde un grand souvenir de Nicolas Perrot. Pendant son séjour dans l'ancienne et historique mission, il a, pendant une quinzaine, exposé à la vénération des fidèles, l'ostensoir donné jadis à la miszion par Nicolas Perrot, lequel ostensoir serait actuellement dans un musée de Chicago.

L. G. 